\title{
Lower lobe origin is a poor prognostic factor in locally advanced non-small-cell lung cancer patients treated with induction chemoradiotherapy
}

\author{
KAZUHIKO SHIEN ${ }^{1,2}$, SHINICHI TOYOOKA ${ }^{1,2}$, JUNICHI SOH ${ }^{1}$, KATSUYUKI HOTTA ${ }^{3}$, KUNIAKI KATSUI ${ }^{4}$, \\ TAKAHIRO OTO $^{1}$, SUSUMU KANAZAWA ${ }^{4}$, KATSUYUKI KIURA ${ }^{3}$, HIROSHI DATE $^{5}$ and SHINICHIRO MIYOSHI $^{1}$ \\ Departments of ${ }^{1}$ Thoracic Surgery, ${ }^{2}$ Clinical Genomic Medicine, ${ }^{3}$ Respiratory Medicine and ${ }^{4}$ Radiology, \\ Okayama University Hospital, Okayama, Okayama 700-8558; ${ }^{5}$ Department of Thoracic Surgery, \\ Graduate School of Medicine, Kyoto University, Kyoto, Kyoto 606-8507, Japan
}

Received September 15, 2014; Accepted January 27, 2015

DOI: $10.3892 / \mathrm{mco} .2015 .509$

\begin{abstract}
The aim of this study was to identify prognostic factors in patients receiving trimodality therapy for locally advanced non-small-cell lung cancer (NSCLC). Among patients who underwent induction chemoradiotherapy (CRT) followed by surgery between 1999 and 2011 at our institution, 76 NSCLC patients with clinical (c) N2/3 stage III were enrolled in this retrospective study. Induction CRT consisted of docetaxel and cisplatin with concurrent 40-60 Gy radiation therapy. In total, 76 patients were assessed (53 men and 23 women) with 43 adenocarcinomas and 33 non-adenocarcinomas. Of the 76 patients, 44 had cStage IIIA and 32 had cStage IIIB disease. The primary tumors were located in the right upper lobe $(\mathrm{n}=33)$, right middle lobe $(\mathrm{n}=5)$, right lower lobe $(n=11)$, left upper lobe $(n=20 s)$ and left lower lobe $(n=7)$. For all 76 patients, lower lobe tumors were associated with a significantly shorter overall survival (OS) and disease-free survival (DFS) compared to non-lower lobe tumors (OS, $\mathrm{P}=0.022$; and DFS, $\mathrm{P}=0.0007)$. When the analysis was limited to pathologically proven $\mathrm{N} 2 / 3$ disease prior to induction CRT $(n=36)$, lower lobe location, compared to other locations, tended to be a poor prognostic factor (OS, $\mathrm{P}=0.068$; and $\mathrm{DFS}, \mathrm{P}=0.0075)$. Our results indicated that a lower lobe tumor origin is associated with unfavorable prognosis in NSCLC patients treated with induction CRT, strongly suggesting the significance of appropriate patient selection in order to maximize the benefits of trimodality therapy.
\end{abstract}

Correspondence to: Professor Shinichi Toyooka, Department of Clinical Genomic Medicine, Okayama University Hospital, 2-5-1 Shikata-cho, Okayama, Okayama 700-8558, Japan

E-mail: toyooka@md.okayama-u.ac.jp

Key words: non-small-cell lung cancer, induction chemoradiotherapy, surgery, N2 disease

\section{Introduction}

Trimodality therapy consisting of induction chemoradiotherapy (CRT) followed by surgery is a potential treatment option for locally advanced non-small-cell lung cancer (NSCLC). Two randomized phase III trials investigated the prognostic effect of surgery following induction CRT in patients with mediastinal lymph nodal metastasis $(1,2)$. Although these studies failed to demonstrate a benefit from the addition of surgery in the overall patient population, the subset analysis of the intergroup trial 0139 indicated that surgical resection following induction CRT was beneficial for those who did not undergo pneumonectomy, strongly suggesting the significance of appropriate patient selection (2). Thus, identifying factors for selecting patients likely to benefit from trimodality therapy is crucial for determining the optimal therapeutic strategy.

Clinical factors estimated prior to therapy initiation are potential predictors of poor patient outcome. Among these factors, lower lobe tumor origin was reported by several investigators to be associated with an unfavorable prognosis in surgically treated patients (3-5). However, controversial results, obtained from patients with early-stage NSCLC, suggested that there is no prognostic difference according to tumor location (6).

In this study, we retrospectively investigated the prognostic significance of tumor location in NSCLC patients with clinical (c) $\mathrm{N} 2 / 3$ disease receiving trimodality therapy.

\section{Patients and methods}

Patients. Induction CRT has been used for locally advanced NSCLC in Okayama University Hospital since $1998(7,8)$. Among the 102 NSCLC patients who underwent induction CRT followed by surgery between January, 1999 and November, 2011 at our institution, 76 patients with $\mathrm{cN} 2 / 3$ stage III disease were enrolled in this retrospective study. The medical records of the NSCLC patients who had undergone induction CRT followed by surgery were reviewed. Staging was performed according to the International Association of the Study of Lung Cancer TNM staging system for NSCLC, 7th edition (9). Disease stage 
was determined using chest radiography, enhanced chest and abdominal computed tomography (CT) scans, enhanced brain magnetic resonance imaging (MRI), radionuclide bone scan or 18-fluoro-2-deoxyglucose positron emission tomography-CT scan and bronchoscopy. The regional mediastinal lymph nodes for each lobe were defined as follows: Right upper lobe (RUL) for superior mediastinal nodes, right middle lobe (RML) for superior mediastinal and subcarinal nodes, right lower lobe (RLL) for subcarinal and inferior mediastinal nodes, left upper lobe (LUL) for superior mediastinal nodes and left lower lobe (LLL) for subcarinal and inferior mediastinal nodes. Metastatic lymph nodes extending over regional nodes were defined as beyond regional. Staging cervical mediastinoscopy was undertaken in a proportion of patients to evaluate bilateral node stations. 2 and 4 and subcarinal station 7 .

This study was approved by the Institutional Review Board/Ethics Committee of Okayama University, Okayama, Japan.

Induction therapy, surgery and adjuvant treatment. Induction CRT was performed as previously described (7). Briefly, docetaxel $\left(40 \mathrm{mg} / \mathrm{m}^{2}\right)$ was administered intravenously followed by cisplatin $\left(40 \mathrm{mg} / \mathrm{m}^{2}\right)$ prior to radiotherapy on days 1 and 8 . Chemotherapy was repeated with a 3- or 4-week interval. Radiotherapy was initiated on the first day of chemotherapy using a 6-10 MV linear accelerator. A total radiation dose of 40-60 Gy was planned using a conventional fractionation protocol ( $2 \mathrm{~Gy} /$ day). The original volume included the primary tumor site, with a margin of $2 \mathrm{~cm}$ around the mass, the ipsilateral hilum and the entire width of the mediastinum, with a margin of $1 \mathrm{~cm}$ around the radiographically visible region of involvement, extending inferiorly to $2 \mathrm{~cm}$ below the carina or $2 \mathrm{~cm}$ below the radiographically identified tumor mass. Following induction CRT, the patients were evaluated for response to treatment. Patients without progressive disease (PD) and/or in good general condition proceeded to undergo surgery.

The surgical procedure was determined based on the extent of the disease prior to induction therapy. Resection with reconstruction of the chest wall or major vessels was performed when necessary. The bronchial stump was covered with pericardial fat tissue or an intercostal muscle pedicle. When a sleeve resection was performed, the greater omentum was used to wrap the anastomosis. Although posterolateral thoracotomy was the basic approach, a median sternotomy or a trap-door approach was employed for patients with supraclavicular or contralateral mediastinal lymph node metastasis, or when securing great vessels, such as the main pulmonary artery, was required to ensure safe resection. Complete ipsilateral superior mediastinal and subcarinal lymph node dissection was routinely undertaken. For patients with primary lower lobe lesions, the lymph nodes of stations 8 and 9 were also resected. Postoperative treatment was left to the primary physician's discretion.

Estimation. Radiological response was assessed using the Eastern Cooperative Oncology Group (ECOG) criteria with certain modifications, as previously reported, and was classified as complete response (CR), partial response (PR), stable disease (SD) and PD (7,10). The anastomotic complications included bronchopleural or bronchovascular fistula, bleeding, bronchial stenosis and malacia. As part of the routine follow-up care, chest and abdominal CT and enhanced brain MRI examination were repeated every 3 months for at least the first 2 years and every 6 months during the 3-5 years following completion of the trimodality therapy.

Statistical analysis. The overall survival (OS) and disease-free survival (DFS) were calculated from the date of the initiation of induction CRT until the date of death or the last follow-up for OS and until confirmed death from any cause or disease recurrence at a local or distant site for DFS. Locoregional recurrence was defined as that developing in the ipsilateral chest or mediastinum and distant recurrence as that developing in any other location.

The Wilcoxon rank-sum test and the Fisher's exact test were used to compare the differences between two groups, as appropriate. A univariate analysis of OS and DFS was performed using the Kaplan-Meier method with the log-rank test. All the data were analyzed using JMP software, version 9.0.0 (SAS Institute Inc., Cary, NC, USA). The statistical tests were two-sided and $\mathrm{P}<0.05$ was defined as indicating statistically significant differences.

\section{Results}

Patient characteristics. Between January, 1999 and November, 2011, a total of 102 NSCLC patients underwent surgery following CRT at the Okayama University Hospital. Patients with Pancoast tumors or $\mathrm{cN} 0 / 1$ disease were excluded. As a result, 76 patients with $\mathrm{cN} 2 / 3$ disease were enrolled in this retrospective study. The patient characteristics are summarized in Table I. The median patient age was 60 years (range, 31-76 years). The 76 patients included 53 men and 23 women, 43 with adenocarcinomas and 33 with non-adenocarcinomas. Of the 76 patients, 44 had stage IIIA and 32 had stage IIIB disease. The primary tumors were located in the RUL $(n=33)$, RML ( $n=5)$, RLL $(n=11)$, LUL $(n=20)$ and LLL (7 patients). Mediastinal lymph node metastasis was pathologically confirmed in 36 patients by mediastinoscopy or endobronchial ultrasound-guided transbronchial biopsy prior to induction CRT.

Trimodality therapy. Of the 76 patients, 49 had completed the planned induction CRT without dose reduction of chemotherapy or radiotherapy. The toxicities were similar to those previously described (7). The median radiation dose was $46 \mathrm{~Gy}$. The radiological response was $\mathrm{CR}$ in 1 patient $(1.3 \%)$, PR in 35 (46.1\%) and SD in 40 (52.6\%), whereas there were no reported cases of PD (Table I). Lobectomy was performed in 54 patients, sleeve lobectomy in 10, bilobectomy in 7 and pneumonectomy in 5 patients. All the patients underwent complete resection, with a negative bronchial margin confirmed by frozen section. The median postoperative hospital stay was 23 days. Severe postoperative pulmonary complications, including anastomotic complications and empyema, occurred in 4 patients. There was no reported trimodality treatment-related mortality.

Lobe-specific treatment response and survival. The patient characteristics and response to treatment in patients with 
Table I. Patient characteristics.

\begin{tabular}{|c|c|c|c|c|}
\hline Variables & $\begin{array}{l}\text { Patient no. } \\
\qquad(\mathrm{n}=76)\end{array}$ & $\begin{array}{c}\text { Non-lower lobe } \\
\quad(\mathrm{n}=58)\end{array}$ & $\begin{array}{l}\text { Lower lobe } \\
\qquad(\mathrm{n}=18)\end{array}$ & P-value \\
\hline Median age (years), range & $60,31-76$ & $60,31-76$ & $60,43-74$ & 0.47 \\
\hline \multicolumn{5}{|l|}{ Gender } \\
\hline Male/female & $53 / 23$ & $41 / 17$ & $12 / 6$ & 0.77 \\
\hline Smoking history & & & & 0.10 \\
\hline Non-smoker/smoker & $17 / 59$ & $10 / 48$ & $7 / 11$ & \\
\hline \multicolumn{5}{|l|}{ Histology } \\
\hline $\mathrm{Ad} / \mathrm{Sq} / \mathrm{AdSq} / \mathrm{LC}$ & $43 / 30 / 1 / 2$ & $33 / 22 / 1 / 2$ & $10 / 8 / 0 / 0$ & 0.78 \\
\hline \multicolumn{5}{|l|}{ Tumor location } \\
\hline RUL/RML/RLL/LUL/LLL & $33 / 5 / 11 / 20 / 7$ & - & - & - \\
\hline \multicolumn{5}{|l|}{$\mathrm{cN}$} \\
\hline $\mathrm{N} 2 / \mathrm{N} 3$ & $66 / 10$ & $49 / 9$ & $17 / 1$ & 0.44 \\
\hline \multicolumn{5}{|l|}{$\mathrm{cN} 2 / 3$} \\
\hline Single/multiple stations & $43 / 33$ & $33 / 25$ & $10 / 8$ & 1.00 \\
\hline \multicolumn{5}{|l|}{$\mathrm{cN} 2 / 3$} \\
\hline Regional/beyond regional nodes & $53 / 23$ & $46 / 12$ & $7 / 11$ & $0.0016^{\mathrm{a}}$ \\
\hline \multicolumn{5}{|l|}{ cStage } \\
\hline IIIA/IIIB & $44 / 32$ & $30 / 28$ & $14 / 4$ & 0.060 \\
\hline Median radiation dose (Gy), range & $46,18-63$ & $46,30-63$ & $46,8-54$ & 0.42 \\
\hline \multicolumn{5}{|l|}{ Completion of induction treatment } \\
\hline Yes/no & $49 / 27$ & $36 / 22$ & $13 / 5$ & 0.58 \\
\hline \multicolumn{5}{|l|}{ Radiological response } \\
\hline $\mathrm{CR} / \mathrm{PR} / \mathrm{SD} / \mathrm{PD}$ & $1 / 35 / 40 / 0$ & $1 / 26 / 31 / 0$ & $0 / 9 / 9 / 0$ & 0.72 \\
\hline \multicolumn{5}{|l|}{ Operation } \\
\hline Not pneumonectomy/pneumonectomy & $71 / 5$ & $54 / 4$ & $17 / 1$ & 1.00 \\
\hline \multicolumn{5}{|l|}{ Severe postoperative complications ${ }^{\mathrm{b}}$} \\
\hline Yes/no & $4 / 72$ & $2 / 56$ & $2 / 16$ & 0.24 \\
\hline \multicolumn{5}{|l|}{ Pathological response (primary lesion) ${ }^{\mathrm{c}}$} \\
\hline $0 / 1 / 2 / 3$ & $1 / 14 / 30 / 31$ & $1 / 8 / 25 / 24$ & $0 / 6 / 5 / 7$ & 0.22 \\
\hline \multicolumn{5}{|l|}{$\mathrm{pN}$} \\
\hline $0 / 1 / 2 / 3$ & $44 / 3 / 23 / 6$ & $35 / 2 / 19 / 2$ & $9 / 1 / 4 / 4$ & 0.12 \\
\hline \multicolumn{5}{|l|}{$\mathrm{pCR}$} \\
\hline Yes/no & $22 / 54$ & $18 / 40$ & $4 / 14$ & 0.56 \\
\hline \multicolumn{5}{|l|}{ Adjuvant therapy } \\
\hline Yes/no & $30 / 46$ & $25 / 33$ & $5 / 13$ & 0.28 \\
\hline \multicolumn{5}{|l|}{ Recurrence } \\
\hline Yes/no & $32 / 44$ & $19 / 39$ & $13 / 5$ & $0.0054^{\mathrm{a}}$ \\
\hline
\end{tabular}

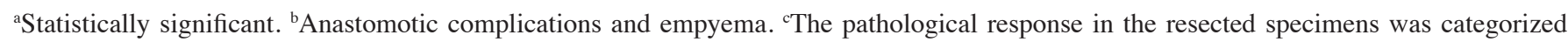
as follows: 0 , no response; $1,>1 / 3$ of cancer cells viable; $2,<1 / 3$ of cancer cells viable; and 3 , no viable cancer cells. Ad, adenocarcinoma; Sq, squamous cell carcinoma; AdSq, adenosquamous cell carcinoma; LC, large-cell carcinoma; RUL, right upper lobe; RML, right middle lobe; RLL, right lower lobe; LUL, left upper lobe; LLL, left lower lobe; CR, complete response; PR, partial response; SD, stable disease; PD, progressive disease; c, clinical; $\mathrm{p}$, pathological.

non-lower lobe and lower lobe tumors are presented in Table I. There was a significant difference in the incidence of beyond regional nodal metastasis between non-lower lobe and lower lobe tumors. Lower lobe tumors were associated with a higher incidence of beyond regional nodal metastasis compare to non-lower lobe tumors (61.1 vs. $20.7 \%$, respectively; $\mathrm{P}=0.0016)$. Radiological responses (CR or $\mathrm{PR}$ ) were documented in $27(46.6 \%)$ of the patients with non-lower 
Table II. Prognostic factors.

\begin{tabular}{|c|c|c|c|c|c|}
\hline Variables & $\begin{array}{l}\text { Patient no. } \\
\quad(\mathrm{n}=76)\end{array}$ & $\begin{array}{c}5 \text {-year } \\
\text { OS rate }(\%)\end{array}$ & P-value & $\begin{array}{c}\text { 5-year } \\
\text { DFS rate }(\%)\end{array}$ & P-value \\
\hline \multicolumn{6}{|l|}{ Pretreatment variables } \\
\hline \multicolumn{6}{|l|}{ Age (years) } \\
\hline \multicolumn{6}{|l|}{ Gender } \\
\hline Male/female & $53 / 23$ & $67.0 / 71.7$ & 0.35 & $57.9 / 43.9$ & 0.20 \\
\hline \multicolumn{5}{|l|}{ Smoking history } & 0.15 \\
\hline \multicolumn{5}{|l|}{ Histology } & 0.19 \\
\hline \multicolumn{6}{|l|}{ Tumor location } \\
\hline \multicolumn{6}{|l|}{$\mathrm{cN}$} \\
\hline $2 / 3$ & $66 / 10$ & $72.6 / 49.2$ & 0.075 & $59.0 / 14.6$ & $0.050^{\mathrm{a}}$ \\
\hline \multicolumn{6}{|l|}{$\mathrm{c}-\mathrm{N} 2 / 3$} \\
\hline $\begin{array}{l}\text { Single/multiple stations } \\
\mathrm{cN} 2 / 3\end{array}$ & $43 / 33$ & $79.2 / 65.0$ & 0.18 & $63.1 / 41.3$ & 0.055 \\
\hline Regional/beyond regional nodes & $53 / 23$ & $77.7 / 48.9$ & $0.037^{\mathrm{a}}$ & $68.0 / 18.6$ & $0.0014^{\mathrm{a}}$ \\
\hline $\begin{array}{l}\text { cStage } \\
\text { IIIA/IIIB }\end{array}$ & $44 / 32$ & $72.1 / 64.9$ & 0.17 & $50.6 / 57.0$ & 0.74 \\
\hline \multicolumn{6}{|l|}{ Treatment-related variables } \\
\hline $\begin{array}{l}\text { Completion of induction CRT } \\
\text { Yes/no }\end{array}$ & $49 / 27$ & $71.3 / 66.5$ & 0.38 & $53.2 / 54.6$ & 0.92 \\
\hline \multicolumn{6}{|l|}{ Radiological response } \\
\hline \multicolumn{6}{|l|}{ Operation } \\
\hline \multicolumn{6}{|l|}{ Severe postoperative complications ${ }^{\mathrm{b}}$} \\
\hline \multicolumn{5}{|l|}{ Pathological response (primary lesion) ${ }^{\mathrm{c}}$} & 0.29 \\
\hline \multicolumn{6}{|l|}{$\mathrm{pN}$} \\
\hline \multicolumn{6}{|l|}{$\mathrm{pCR}$} \\
\hline Yes/no & $22 / 54$ & $80.5 / 64.5$ & 0.72 & $62.2 / 49.1$ & 0.64 \\
\hline \multicolumn{6}{|l|}{ Adjuvant therapy } \\
\hline Yes/no & $30 / 46$ & $69.1 / 71.5$ & 0.62 & $44.8 / 60.6$ & 0.54 \\
\hline
\end{tabular}

${ }^{a}$ Statistically significant. ${ }^{\mathrm{b}}$ Anastomotic complications and empyema. ${ }^{\mathrm{c}}$ The pathological response in the resected specimens was categorized as follows: 0 , no response; $1,>1 / 3$ of cancer cells viable; $2,<1 / 3$ of cancer cells viable; and 3 , no viable cancer cells. OS, overall survival; DFS, diseas-free survival; Ad, adenocarcinoma; CRT, chemoradiotherapy; CR, complete response; PR, partial response; SD, stable disease; NR, not reached; c, clinical; p, pathological.

lobe and $9(50.0 \%)$ of those with lower lobe tumors $(\mathrm{P}=0.72)$. Severe postoperative complications, including empyema and anastomotic complications, occurred in 2 patients $(3.4 \%)$ with non-lower lobe and $2(1.1 \%)$ with lower lobe tumors $(\mathrm{P}=0.24)$. Adjuvant treatment was administered to 25 patients $(43.1 \%)$ with non-lower lobe and $5(27.8 \%)$ with lower lobe tumors $(\mathrm{P}=0.28)$.

At the time of data analysis in June, 2013, the 5-year OS and DFS were 69.4 and $53.5 \%$, respectively. The median follow-up period was 64 months. To clarify pre- and 
Table III. Prognostic factors limited to pathologically proven N2/3 disease.

\begin{tabular}{|c|c|c|c|c|c|}
\hline Variables & $\begin{array}{l}\text { Patient no. } \\
\qquad(\mathrm{n}=36)\end{array}$ & $\begin{array}{l}5 \text {-year } \\
\text { OS rate }(\%)\end{array}$ & P-value & $\begin{array}{c}\text { 5-year } \\
\text { DFS rate }(\%)\end{array}$ & P-value \\
\hline \multicolumn{6}{|l|}{ Pretreatment variables } \\
\hline \multicolumn{6}{|l|}{ Age (years) } \\
\hline \multicolumn{6}{|l|}{ Gender } \\
\hline Male/female & $24 / 12$ & $66.1 / 80.0$ & 0.18 & $55.7 / 58.3$ & 0.95 \\
\hline \multicolumn{5}{|l|}{ Smoking history } & 0.72 \\
\hline \multicolumn{4}{|l|}{ Histology } & $36.9 / 76.0$ & 0.12 \\
\hline \multicolumn{6}{|l|}{ Tumor location } \\
\hline \multicolumn{6}{|l|}{$\mathrm{cN}$} \\
\hline $2 / 3$ & $33 / 3$ & $69.7 / 100$ & 0.38 & $55.8 / 50.0$ & 0.64 \\
\hline \multicolumn{6}{|l|}{$\mathrm{cN} 2 / 3$} \\
\hline $\begin{array}{l}\text { Single/multiple stations } \\
\mathrm{cN} 2 / 3\end{array}$ & $24 / 12$ & $82.6 / 52.1$ & 0.096 & $65.4 / 33.8$ & 0.12 \\
\hline Regional/beyond regional nodes & $29 / 7$ & $77.1 / 47.6$ & 0.37 & $64.1 / \mathrm{NR}$ & 0.097 \\
\hline $\begin{array}{l}\text { cStage } \\
\text { IIIA/IIIB }\end{array}$ & $27 / 9$ & $67.0 / 85.7$ & 0.76 & $53.0 / 64.8$ & 0.75 \\
\hline \multicolumn{6}{|l|}{ Treatment-related variables } \\
\hline $\begin{array}{l}\text { Completion of induction CRT } \\
\text { Yes/no }\end{array}$ & $22 / 14$ & $74.4 / 68.8$ & 0.43 & $53.2 / 60.1$ & 0.85 \\
\hline \multicolumn{6}{|l|}{ Radiological response } \\
\hline \multicolumn{6}{|l|}{ Operation } \\
\hline \multicolumn{6}{|l|}{ Severe postoperative complications ${ }^{\mathrm{b}}$} \\
\hline $\begin{array}{l}\text { Pathological response (primary lesion) } \\
\text { 3/0-2 }\end{array}$ & $12 / 24$ & $83.3 / 65.4$ & 0.62 & $40.4 / 83.3$ & 0.10 \\
\hline \multicolumn{5}{|l|}{$\mathrm{pN}$} & 0.71 \\
\hline \multicolumn{5}{|l|}{$\mathrm{pCR}$} & 0.31 \\
\hline \multicolumn{6}{|l|}{ Adjuvant therapy } \\
\hline Yes/no & $16 / 20$ & $68.6 / 49.8$ & 0.73 & $43.8 / 64.6$ & 0.80 \\
\hline
\end{tabular}

${ }^{a}$ Statistically significant. ${ }^{\mathrm{b}}$ Anastomotic complications and empyema. ${ }^{\mathrm{C}}$ The pathological response in the resected specimens was categorized as follows: 0 , no response; $1,>1 / 3$ of cancer cells viable; $2,<1 / 3$ of cancer cells viable; and 3 , no viable cancer cells. OS, overall survival; DFS, diseas-free survival; Ad, adenocarcinoma; CRT, chemoradiotherapy; CR, complete response; PR, partial response; SD, stable disease; NR, not reached; c, clinical; p, pathological.

post-treatment prognostic factors, we separately analyzed pretreatment and treatment-related prognostic factors. The significant pretreatment prognostic factor for OS were found to be tumor location and presence of beyond regional nodal metastases. The significant prognostic factors for DFS were tumor location, $\mathrm{cN}$ stage and presence of beyond regional nodal metastases (Table II). Among the treatment-related factors, there was no significant prognostic factor for OS, whereas radiological response was the only significant prognostic factor for DFS. 


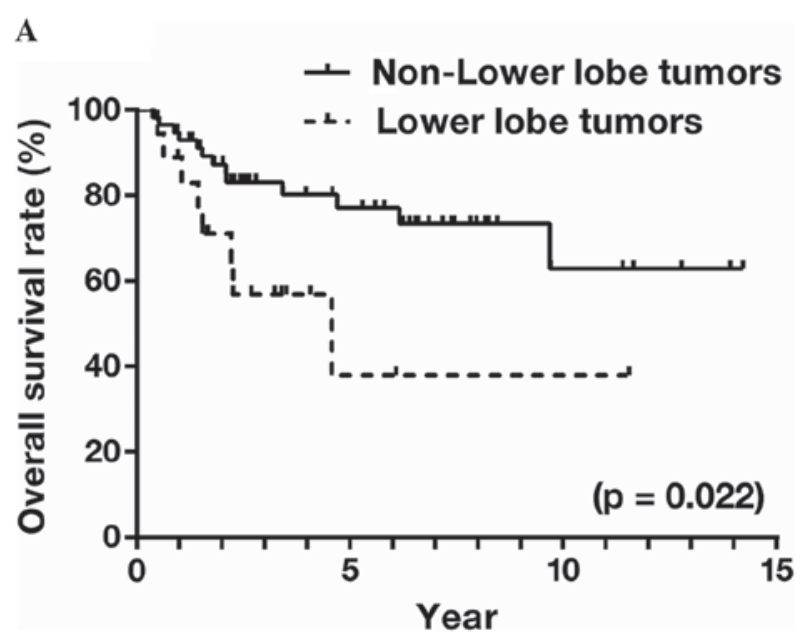

B

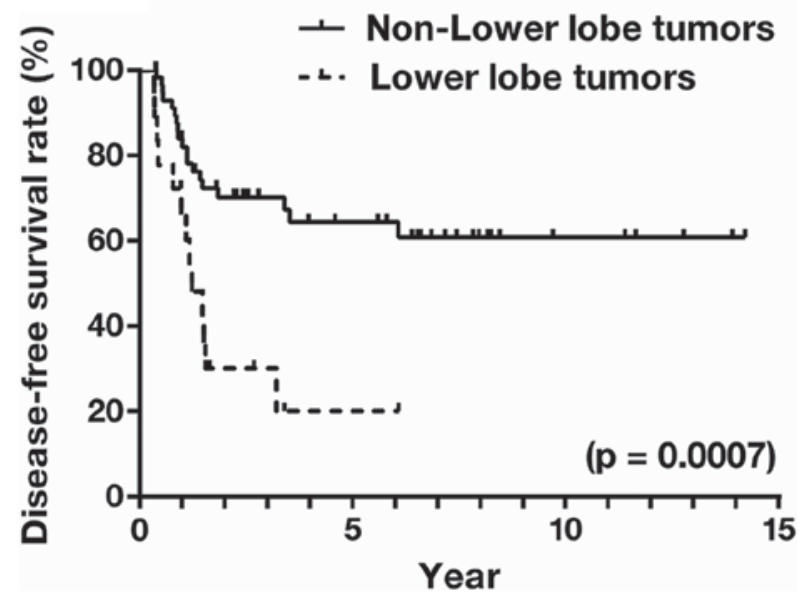

Figure 1. (A) Overall and (B) disease-free survival curves stratified by tumor location.

For the entire patient group, lower lobe tumors were associated with significantly shorter OS and DFS compared to other locations (OS, $\mathrm{P}=0.022$; and $\mathrm{DFS}, \mathrm{P}=0.0007$; Fig. $1 \mathrm{~A}$ and $\mathrm{B}$ ). The respective 5-year OS and DFS were 77.0 and $64.4 \%$ for non-lower lobe tumors and 37.9 and $20.1 \%$ for lower lobe tumors. The disease relapsed as distant metastasis in 14 and locoregional recurrence in 5 patients with non-lower lobe tumors and as distant metastasis in 11 and locoregional recurrence in 2 patients with lower lobe tumors $(\mathrm{P}=0.67)$.

When the analysis was limited to pathologically proven $\mathrm{N} 2 / 3$ disease prior to induction CRT $(n=36)$, patients with lower lobe tumors tended to have an unfavorable OS $(\mathrm{P}=0.068)$ and DFS ( $\mathrm{P}=0.0075)$ compared to those with non-lower lobe tumors (Table III). The respective 5-year OS and DFS were 80.4 and $66.0 \%$ in patients with non-lower lobe tumors and 38.1 and $14.3 \%$ in those with lower lobe tumors.

\section{Discussion}

In this study, we demonstrated that tumors arising from the lower lobe were associated with a poor prognosis in patients with locally advanced NSCLC treated with trimodality therapy. The patient characteristics were similar between the two groups and there were no significant differences in the effect of induction CRT between patients with non-lower lobe tumors and those with a lower lobe tumor origin.

One possible explanation for our results is that, at the time of diagnosis, NSCLC patients with tumors arising from the lower lobe had a more extensive disease than expected. Rocha et al (11) demonstrated in their prospective cohort study of 109 NSCLC patients that a lower lobe location of the primary tumor was significantly associated with upstaging following surgery for early-stage (cStage I/II) NSCLC. Kudo et al (5) also reported in their retrospective cohort study of 978 NSCLC patients that the OS rates were similar between patients with LLL and those with non-LLL tumors, regardless of stage, although the 5-year OS rates were significantly poorer for LLL tumors compared to non-left lower tumors among patients with lymph node metastasis. Indeed, our results demonstrated that lower lobe tumors were associated with a significantly higher incidence of beyond regional nodal metastases compared to non-lower lobe tumors. In addition, it has been reported that LLL tumors anatomically tend to metastasize to the contralateral mediastinal nodes via subcarinal nodes more readily compared to RLL tumors (12). Considering these observations, the higher rate of lymph node metastasis from lower lobe tumors possibly contributes to the unfavorable prognosis in patients with such tumors among the patient population with the same cStage, based on radiographic evaluation. There was no significant difference in OS and DFS between tumors originating in the right or left lower lobe. Our findings, along with those of previous studies, also suggest that tumor location may be a prognostic factor and it should be included as a stratification factor in the design of randomized studies, such as the intergroup trial 0139. Imbalanced distribution of tumor location in each arm may significantly affect the outcome of treatment in randomized studies.

There was no statistically significant difference in the effects of induction CRT between non-lower lobe and lower lobe tumors, although the latter were associated with a poorer pathological CR rate (lower lobe vs. non-lower lobe tumors, 22.2 vs. $31.0 \%$ ). Lower lobe tumors may generally be more affected by respiratory movement during radiotherapy compared to non-lower lobe tumors and the radiation fields may be wider in lower lobe compared to those in non-lower lobe tumors (13). Although the exact reason is not known, the difference in respiratory movement during radiation may be responsible for the poor prognosis of patients with lower lobe tumors.

The limitations of this study include its retrospective nature and the small sample size, suggesting that a significant selection bias may be present. In addition, N2/3 stage was not confirmed pathologically prior to induction CRT and certain cases were overestimated as advanced-stage. Therefore, we performed an analysis limited to the 36 cases of pathologically proven N2/3 disease. There was a tendency of poor survival in patients with lower lobe tumors compared to those with non-lower lobe tumors.

In conclusion, tumors arising from the lower lobes of the lungs were found to be a poor prognostic factor in NSCLC patients with N2/3 disease receiving trimodality therapy. Our results suggest that, when designing randomized studies for 
locally advanced NSCLC with mediastinal metastasis, particularly when trimodality therapy is included in the protocol, the tumor location should be considered as a stratification factor.

\section{Acknowledgements}

Katsuyuki Hotta has received honoraria from Chugai Pharmaceutical, Eli Lilly Japan and Pfizer. Katsuyuki Kiura has received honoraria from AstraZeneca, Chugai Pharmaceutical, Daiichi Sankyo, Eli Lily, GlaxoSmithKline, Novartis, Sanofi-Aventis and Taiho Pharmaceutical.

\section{References}

1. van Meerbeeck JP, Kramer GW, Van Schil PE, et al: Randomized controlled trial of resection versus radiotherapy after induction chemotherapy in stage IIIA-N2 non-small-cell lung cancer. J Natl Cancer Inst 99: 442-450, 2007.

2. Albain KS, Swann RS, Rusch VW, et al: Radiotherapy plus chemotherapy with or without surgical resection for stage III non-small-cell lung cancer: a phase III randomised controlled trial. Lancet 374: 379-386, 2009.

3. Ichinose Y, Kato H, Koike T, et al: Completely resected stage IIIA non-small cell lung cancer: the significance of primary tumor location and N2 station. J Thorac Cardiovasc Surg 122: 803-808, 2001.

4. Ou SH, Zell JA, Ziogas A and Anton-Culver H: Prognostic factors for survival of stage I nonsmall cell lung cancer patients: a population-based analysis of 19,702 stage I patients in the California Cancer Registry from 1989 to 2003. Cancer 110: 1532-1541, 2007.
5. Kudo Y, Saji H, Shimada Y, et al: Do tumours located in the left lower lobe have worse outcomes in lymph node-positive non-small cell lung cancer than tumours in other lobes? Eur J Cardiothorac Surg 42: 414-419, 2012.

6. Puri V, Garg N, Engelhardt EE, et al: Tumor location is not an independent prognostic factor in early stage non-small cell lung cancer. Ann Thorac Surg 89: 1053-1059, 2010.

7. Katayama H, Ueoka H, Kiura K, et al: Preoperative concurrent chemoradiotherapy with cisplatin and docetaxel in patients with locally advanced non-small-cell lung cancer. Br J Cancer 90: 979-984, 2004.

8. Toyooka S, Kiura K, Shien K, et al: Induction chemoradiotherapy is superior to induction chemotherapy for the survival of non-small-cell lung cancer patients with pathological mediastinal lymph node metastasis. Interact Cardiovasc Thorac Surg 15: 954-960, 2012.

9. Goldstraw P, Crowley J, Chansky K, et al; International Association for the Study of Lung Cancer International Staging Committee; Participating Institutions: The IASLC Lung Cancer Staging Project: proposals for the revision of the TNM stage groupings in the forthcoming (seventh) edition of the TNM Classification of malignant tumours. J Thorac Oncol 2: 706-714, 2007.

10. Oken MM, Creech RH, Tormey DC, et al: Toxicity and response criteria of the Eastern Cooperative Oncology Group. Am J Clin Oncol 5: 649-655, 1982.

11. Rocha AT, McCormack M, Montana G and Schreiber G: Association between lower lobe location and upstaging for early-stage non-small cell lung cancer. Chest 125: 1424-1430, 2004.

12. Nohl-Oser HC: An investigation of the anatomy of the lymphatic drainage of the lungs as shown by the lymphatic spread of bronchial carcinoma. Ann R Coll Surg Engl 51: 157-176, 1972.

13. Hayakawa K, Mitsuhashi N, Saito Y, et al: Impact of tumor extent and location on treatment outcome in patients with stage III non-small cell lung cancer treated with radiation therapy. Jpn J Clin Oncol 26: 221-228, 1996. 